\title{
Quality of Life after Surgery for Stanford Type A Aortic Dissection: Influences of Different Operative Strategies
}

\author{
Tamer Ghazy, ${ }^{1}$ Mohamed Eraqi,${ }^{1}$ Adrian Mahlmann, ${ }^{2}$ Helena Hegelmann, ${ }^{2}$ Klaus Matschke, ${ }^{1}$ Utz Kappert, ${ }^{1}$ \\ Norbert Weiss ${ }^{2}$
}

${ }^{1}$ Department of Cardiac Surgery of the Dresden Heart Centre, ${ }^{2}$ UniversitätsGefäßCentrum, Dresden University of Technology, Dresden, Germany

\section{ABSTRACT}

Background: Outcome of surgery for acute Stanford type A aortic dissection extends beyond mortality and morbidity. More than one operative strategy is available but little is known regarding their influence on quality of life. This study analyzes the influence of defensive and aggressive operative strategies on the patients' midterm quality of life (QoL).

Methods: From July 2007 to July 2010, 95 patients underwent surgery for acute Stanford type A aortic dissection in our institution. Patients who survived the procedure, gave consent to inclusion in the institution prospective registry, completed at least 2-years of follow-up protocol, and answered two quality of life questionnaires (SF-36 and WHO-QOL-BREF) were included in the study. Patients were divided into two groups according to operative strategy: defensive (DS) with replacement of the ascending aorta only, and aggressive (AS) with replacement of the ascending aorta, aortic arch with/out a frozen elephant trunk procedure. The preoperative, operative, postoperative and the midterm QoL were analyzed and compared.

Results: 39 patients were included in the study. The DS group had a shorter operative time $(184 \pm 54$ versus $276 \pm$ 110 minutes respectively, $P=.001)$. The AS group had higher incidence of dialysis (31\% versus $4 \%$ respectively, $P=.038$ ). The midterm QoL analysis showed a collective lower value than the normal population. In the SF-36, DS performed better in all categories but with no statistical significance. In the WHO-QOL-BREF, DS performed significantly better in the global life quality and psychological health categories $(P=.038$ and .049 respectively).

Conclusion: In Stanford type A aortic dissection, adopting an aggressive surgical strategy does not improve the quality of life in midterm follow-up compared to a defensive strategy. Unless the clinical setting dictates an aggressive management strategy, a defensive strategy can be safely adopted.

\section{INTRODUCTION}

For many years, morbidity and mortality have been considered the key markers of surgical outcome and have routinely been used to assess performance and evaluate new

Received December 9, 2016; accepted fanuary 14, 2017.

Correspondence: Dr: med. Tamer Ghazy, Dresden Heart Center University Hospital, Fetscherstrasse 76, 01307 Dresden, Germany; +49-351-4501511; fax: +49-351-4501512 (e-mail: tamer_ghazy@hotmail.com). procedures. With continuing efforts to achieve patient-centered health care, however, it has become apparent that more subjective measures, such as quality of life (QoL), health status (HS), and patient satisfaction (PS) are also of equal importance [George 2012].

There are several reasons why there is a great interest in measuring the outcomes of healthcare. Knowing how a surgical intervention affects a disease process or a patient's health can help determine whether it is effective, as comparing treatment outcomes may indicate the extent to which one intervention is better than another [David 2005]. Furthermore, both qualitative and quantitative measurements provide important information for patients about the expected outcome of their treatment.

Aside from morbidity and mortality rates, which are widely published, there is little information about QoL among patients who undergo Stanford type A aortic dissection surgery. That is why evidence-based data on postoperative QoL in this patient cohort are needed. The aim of the presented study is to compare the patients' postoperative QoL after different operative management strategies for acute Stanford type A aortic dissection, to reveal if aggressive operative management would lead to a better QoL in midterm follow-up.

\section{PATIENTS AND METHODS}

Between July 2007 and July 2010, patients who underwent emergency surgery in our center for acute Stanford type A aortic dissection were screened for inclusion in this study. The inclusion criteria were age over 18 years, consent to inclusion into our institution's prospective aortic registry, surviving the procedure, and undergoing a standardized follow-up protocol according to the university's interdisciplinary board for aortic disease for at least two years. All patients who met the inclusion criteria were included in the study. The study was approved by the university's ethics committee. There was no conflict of interest. No exclusion criteria applied.

\section{Baseline Characteristics}

The baseline characteristics of the patient population were collected through history taking, the preoperative patients' reports, and clinical examination. Operative risk was estimated using the logistic EuroSCORE.

\section{Surgical Approach}

All procedures were performed via brachicephalic arterial cannulation and under moderate hypotheramic circulatory 
Table 1. Baseline Characteristics

\begin{tabular}{|c|c|c|c|c|}
\hline & Collective & DS & AS & $P$ \\
\hline \multicolumn{5}{|l|}{ Patient demographics } \\
\hline No. of patients & 39 & 26 & 13 & \\
\hline Age, y & $61 \pm 13$ & $62 \pm 14$ & $62 \pm 12$ & .919 \\
\hline Male sex, \% & 74 & 73 & 77 & .736 \\
\hline \multicolumn{5}{|l|}{ Risk factors and comorbidities, \% } \\
\hline Diabetes mellitus & 10 & 12 & 8 & .818 \\
\hline Hypertension & 92 & 92 & 92 & .747 \\
\hline Hyperlipidemia & 33 & 31 & 38 & .447 \\
\hline Coronary heart disease & 18 & 12 & 31 & .151 \\
\hline Renal insufficiency & 30 & 72 & 67 & .765 \\
\hline COPD & 8 & 4 & 15 & .253 \\
\hline \multicolumn{5}{|c|}{ Clinical presentation and operative risk } \\
\hline $\begin{array}{l}\text { Aortic valve insufficiency } \\
\text { (grade II or higher), \% }\end{array}$ & 41 & 35 & 54 & .210 \\
\hline $\begin{array}{l}\text { Involvement of supraaortic } \\
\text { branches, \% }\end{array}$ & 33 & 27 & 46 & .940 \\
\hline Peripheral organ ischemia, \% & 23 & 23 & 23 & .661 \\
\hline Neurological deficit, \% & 14 & 16 & 8 & .654 \\
\hline Aortic rupture, \% & 23 & 23 & 23 & .790 \\
\hline Redo procedure, \% & 10 & 8 & 15 & .901 \\
\hline Log. EuroSCORE & $27 \pm 13$ & $28 \pm 10$ & $26 \pm 19$ & .759 \\
\hline
\end{tabular}

DS indicates defensive operative strategy; AS, aggressive operative strategy; COPD, chronic obstructive pulmonary disease; Log. EuroSCORE, logistic European System for Cardiac Operative Risk Evaluation.

arrest $\left(28^{\circ} \mathrm{C}\right)$ with unilateral antegrade selective cerebral perfusion. In all cases, a direct inspection of the aortic arch was performed under circulatory arrest. If an entry tear was identified in the arch, a total arch replacement with/out frozen elephant trunk procedure (according to the localization of the entry tear) was performed. If the main entry tear was localized in the ascending aorta, the surgical strategy differed according to the surgeon, following either a defensive management strategy including isolated replacement of the ascending aorta, or an aggressive one including replacement of the ascending aorta and the aortic arch with/out the frozen elephant trunk procedure. In all cases, an aortic valve repair or replacement was performed if a moderate or severe aortic valve insufficiency was documented by echocardiography. The procedures were performed in a standard manner as thoroughly described in the literature [Reece 2008; Karck 2003].

\section{Follow-up Protocol}

Midterm survival data were collected from direct contact with patients, clinic follow-up records, and information from families or referring physicians. All patients who were alive during the period of our study were invited to participate in
Table 2. Intraoperative and Postoperative Data

\begin{tabular}{lcccc}
\hline & Collective & DS & AS & $P$ \\
\hline Intraoperative data & & & & \\
$\quad$ Operative time & $215 \pm 87$ & $184 \pm 54$ & $276 \pm 110$ & .001 \\
Aortic valve repair, \% & 10 & 12 & 8 & .747 \\
Aortic valve replacement, \% & 31 & 27 & 38 & .745 \\
CABG, \% & 10 & 12 & 8 & .592 \\
Postoperative data & & & & \\
Bleeding (>1000 mL/24h), \% & 16 & 20 & 8 & .083 \\
Rethoracotomy, \% & 5 & 4 & 8 & .889 \\
Reintubation, \% & 5 & 4 & 8 & .889 \\
Neurological deficit, \% & 11 & 8 & 15 & .424 \\
Dialysis, \% & 13 & 4 & 31 & .038 \\
Hospital stay & $16 \pm 11$ & $15 \pm 12$ & $19 \pm 7$ & .277 \\
\hline
\end{tabular}

DS indicates defensive operative strategy; AS, aggressive operative strategy; CABG, coronary artery bypass grafting.

follow-up examinations. These included a medical history, assessment of cardiovascular risk factors and comorbidities, and documentation of current medications, physical examination and imaging studies which were performed according to a standardized protocol.

\section{Quality of Life}

All patients who completed the follow-up protocol for at least two years were asked to answer the Medical Study Outcomes Short Form 36 (SF-36) quality of life questionnaire (German version) and the WHO-QOL-BREF test at the end of the second year of follow up.

The SF-36 questionnaire consists of 36 questions in eight scaled scores, which are the weighted sums of the questions in their section. Each scale is directly transformed into a $0-100$ scale. These sections are designed to measure vitality, physical functioning, bodily pain, general health perception, physical role functioning, emotional role functioning, social role functioning, and mental health [Ellert 1999].

The WHO-QOL-BREF test (German version) was developed by the World Health Organization, exists in different languages, and is used to study the categories "global life quality," "physical," "psychological," "social relations," and "environment" of life quality. It contained 26 items to be answered on a scale of 1-5 [Angermeyer 2000].

\section{Subgroup Analysis and Definitions}

In addition to analysis of the population as a whole, the patient population was divided into two groups according to the operative strategy treating the diseased aorta. The defensive strategy group (DS) was defined as patients who received a replacement of the ascending aorta only. The aggressive strategy group (AS) was defined as patients who received, in addition to the replacement of the ascending aorta, an aortic 
Table 3. SF-36 Survey Results

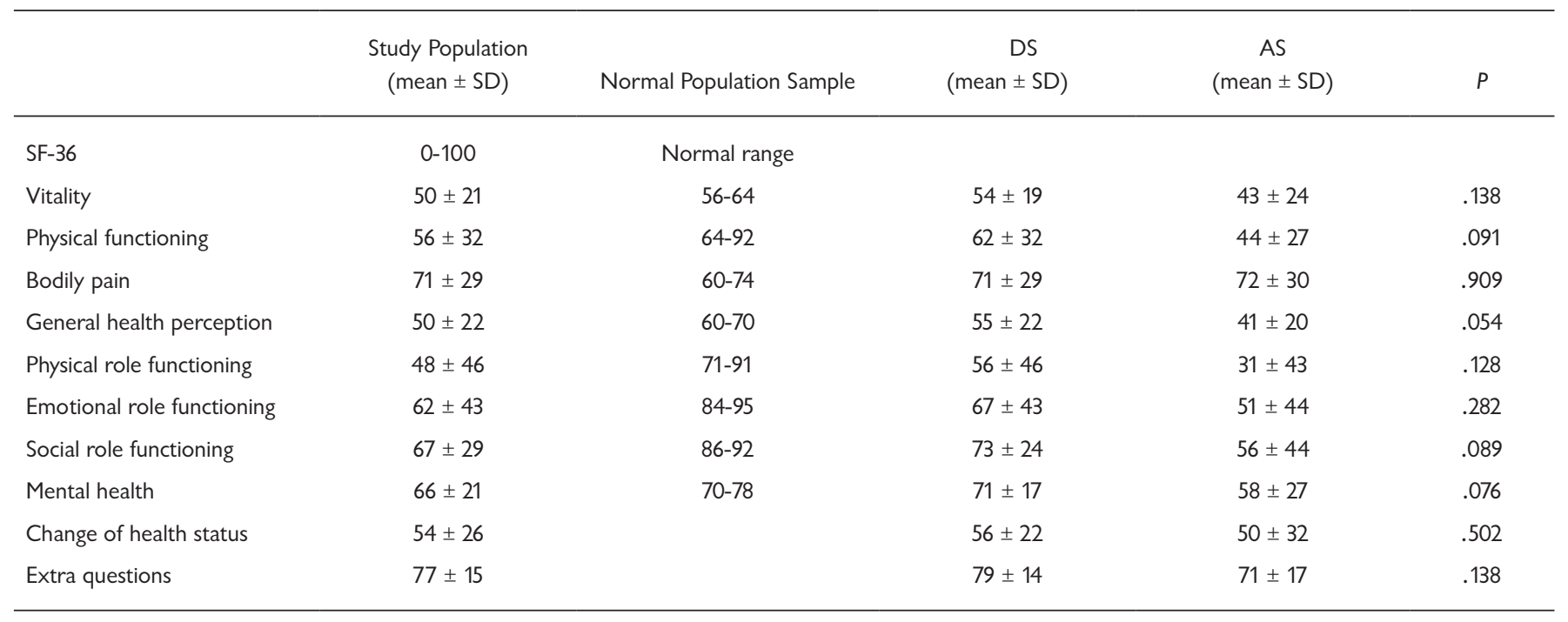

DS indicates defensive operative strategy; AS, aggressive operative strategy.

arch replacement or a frozen elephant trunk procedure. Both groups were then compared in regard to the midterm QoL.

\section{Statistical Analysis}

Continuous variables are presented as mean \pm standard deviation. Binary data are presented as percentage of the total study population. Statistical comparisons regarding the QoL of patients between the groups were made using the Fisher exact test for categorical variables and Student $t$ test for continuous variables. Statistical significance was defined as $\mathrm{P}<$ .05 (two-sided). All statistics were carried out using JMP (version 6.0.0) software (SAS Institute, Cary, NC, USA).

\section{RESULTS}

From 95 patients who underwent surgery for treatment of acute Stanford type A aortic dissection, 39 patients met the inclusion criteria and were included in the study. The DS group included 26 patients and AS group included 13 patients. Baseline characteristics of the patients are presented in Table 1. The patient population consisted of a typical cohort with a logistic EuroSCORE of $27 \pm 13 \% .23 \%$ of patients presented with peripheral organ ischemia, and $23 \%$ with a ruptured aorta. $14 \%$ of patients suffered from neurological deficits at the time of surgery. There were no significant statistical differences in patient characteristics between the two groups (Table 1).

\section{Intra- and Postoperative Results}

The mean operation time was $215 \pm 87$ minutes. The operation time was significantly shorter in DS compared to AS (184 \pm 54 versus $276 \pm 110$ minutes respectively, $P=.001)$. The aortic valve was repaired in $10 \%$ and replaced in $31 \%$ of patients due to aortic insufficiency, with no significant difference between the groups.
The postoperative need for dialysis was higher in AS than DS (31\% versus $4 \%$ respectively, $P=.038)$. Other than that, there were no significant differences between the groups. The detailed intra- and postoperative results are listed in Table 2.

\section{Quality of Life}

SF-36 Questionnaire. The collective results as well as the results pertaining to the two study groups are presented in Table 3. The collective values for bodily pain and mental health were within the normal range for both groups. The midterm achieved values of the other categories were below the reference values of the normal population survey. The physical, emotional, and social role functions' results were the lowest compared to the reference population.

Further analysis revealed that AS patients showed a mental health score below the normal range. Comparing both groups, the DS showed a positive trend in all categories but with no statistical significance.

The WHO-QOL-BREF questionnaire. The analysis of the WHO-QOL-BREF questionnaire revealed a significantly better performance in DS compared to AS regarding the global life quality and psychological health categories $(P=.038$ and .049 respectively). The other categories did not show a significant difference between the groups. The detailed results are listed in Table 4.

\section{DISCUSSION}

Surgery for acute Stanford type A aortic dissection is one of the most challenging clinical settings in adult cardiac surgery. While some surgeons suggest a curative surgical repair with removal and grafting of the whole dissected aortic segments to achieve better mid- and long-term results, other surgeons believe that the acute intervention should be restricted to a life-saving procedure approaching the entry tear only to 
Table 4. WHO-QOL-BREF Survey Results

\begin{tabular}{lcccc}
\hline $\begin{array}{l}\text { WHO-QOL-BREF Study } \\
\text { Population }\end{array}$ & Collective & DS & AS & $P$ \\
\hline Global life quality & $62 \pm 21$ & $67 \pm 20$ & $52 \pm 21$ & .038 \\
Physical health & $65 \pm 22$ & $68 \pm 20$ & $58 \pm 26$ & .191 \\
Psychological health & $73 \pm 16$ & $76 \pm 14$ & $65 \pm 18$ & .049 \\
Social relations & $70 \pm 16$ & $73 \pm 15$ & $63 \pm 16$ & .092 \\
Environment & $77 \pm 11$ & $79 \pm 11$ & $74 \pm 11$ & .253 \\
\hline
\end{tabular}

WHO-QOL-BREF indicates World Health Organization quality of life short questionnaire; DS, defensive operative strategy; AS, aggressive operative strategy.

reduce the operative risk to a minimum to lower the mortality risk [Truls 2007]. The remaining dissection could be addressed in a more controlled setting later on [Uchida 2013].

Most recently, surgeons are increasingly being encouraged to perform patient-oriented analysis of the treatment outcome, driven in large part by healthcare policymakers, payers, and surgical societies [David 2005]. Regardless of the specific reason for measuring surgical outcomes, it is important that the outcomes measured reflect the effects of care in a manner that is meaningful to patients.

As surgical practice evolves, HS and QoL are likely to have increasing significance in relation to management of patients with acute Stanford type A aortic dissection. However, the evidence in this area is yet to evolve. Meticulous follow-up of these patients should include QoL analysis, as these data may help clinicians to determine the optimum operative strategy in the era of evidence-based medicine.

Almost 2300 years ago, the Greek philosopher Aristothe referred to QoL as "eudaimonia" (bliss), and emphasized the subjective nature of the concept [Aristoteles 1993]. Many years later, the World Health Organization (WHO) [WHOQOL 1998] defined quality of life as individuals' perceptions of their position in life, in the context of the cultures and values in the societies in which they live and in relation to their goals, expectations, standards, and concerns [Hanestad 1993; Farquhar 1995].

There is no disease-specific questionnaire for patients with type A acute aortic dissection. In this study, the WHO-QOLBREF test [Angermeyer 2000] was used to measure life quality because of its international comparability and its brevity. The SF-36 was also implemented because it has been suggested as the most useful tool for assessing QoL in vascular patients [George 2012], although it takes longer for patients to answer the questions.

Looking into the results of the study, it became obvious that it was of benefit to use both questionnaires to detect the difference between the influence of AS and DS on the QoL in the midterm. As the SF-36 questionnaire showed a positive trend in all categories in the favor of DS, it did not show a statistical significance. The WHO-QOL-BREF, on the other hand, revealed a significant difference between the groups, again in favor of DS. This is of particular importance in studying Stanford type A aortic dissection, as the low patient volume might limit the statistical power of analyzed data, as seen in this study.

While the protagonists of DS aim to ensure a favorable short-term outcome after surgery for Stanford type A aortic dissection, protagonists of AS aim to improve the mid- and long-term results. As the study revealed, the AS failed to show a better performance regarding the patients' QoL in the midterm. Unless the clinical presentation dictated an AS (eg, a large entry tear in the aortic arch), adopting a DS not only provided a technically easier procedure with favorable short-term outcome, but was also associated with better QoL in the midterm.

A major limitation of this study is the low statistical power due to the small sample size. QoL is a "soft" endpoint that is difficult to evaluate and highly individual [Noyez 2011]. There was no preoperative QoL evaluation, which is very difficult in this type of emergency procedure. Overcoming such obstacles might be possible by increasing the sample size. The best answer to that might be multicenter studies to be able to recruit a larger number of patients in a shorter time.

\section{Conclusion}

In Stanford type A aortic dissection, adopting an aggressive surgical strategy does not improve the quality of life in the midterm compared to a defensive strategy. Unless the clinical setting dictates an aggressive management strategy, a defensive strategy can be safely adopted.

\section{REFERENCES}

Angermeyer MC, Kilian R, Matschinger H. 2000. WHOQOL-100 und WHOQOL-BREF. Handbuch fur die deutschsprachige Version der WHO Instrument zur Erfassung von Lebensqualitat. Gottingen: Hogrefe-Verlag.

Aristoteles. 1993. Ethica Nicomachea A. Athens: Kaktos.

David R. 2005. Measuring quality of life after surgery. Surg Innov 12:161-5.

Ellert U, Bellach M. 1999. Der Bundesgesundheitssurvey - Beschreibung einer aktuellen Normstichprobe. Gesundheitswesen 61:190-4.

Farquhar M. 1995. Definitions of quality of life: a taxonomy. J Adv Nurs 22:502-8.

George P. 2012. Questions remain about quality of life after abdominal aortic aneurysm repair. J Vasc Surg 56:520-7.

Hanestad BR, Albrektsen G. 1993. The effects of participation in a support group on self-assessed quality of life in people with insulin-dependent diabetes mellitus. Diabetes Res Clin Pract 19:163-73.

Karck M, Chavan A, Hagl C, Friedrich H, Galanski M, Haverich A. 2003. The frozen elephant trunk technique: a new treatment for thoracic aortic aneurysms. J Thorac Cardiovasc Surg 125:1550-3.

Noyez L, de Jager MJ, Marku AL. 2011. Quality of life after cardiac surgery: underresearched research. Interact Cardiovasc Thorac Surg 13:511-14.

Reece T, Green G, Kron I. 2008. Aortic Dissection. In: Cohn L, ed. Cardiac surgery in the adult. 3rd ed. New York: McGraw Hill; 1195-1222.

Truls M, Santi T, Vincenzo R. 2007. Cardiovascular surgery in the initial 
treatment of aortic dissection and acute aortic syndroms. In: Baglia R, Nienaber C, Isselbacher E, Eagle K, eds. Aortic dissection and related syndroms. New York: Springer Science + Business Media; 167-90.

Uchida N, Katayama K, Takahashi S, Sueda T. 2013. Endovascular stent grafting of the downstream aorta after complete arch replacement using the frozen elephant trunk technique for acute type A aortic dissection. Eur J Cardiovasc Surg 43:196.

World Health Organization Quality of Life Assessment (WHOQOL). 1998. Development and general psychometric properties. Soc Sci Med 46:1569-85. 